\title{
Intermédialités
}

Histoire et théorie des arts, des lettres et des techniques

Intermediality

History and Theory of the Arts, Literature and Technologies

\section{Bergson anachronique, ou la métaphysique est-elle soluble dans l'intermédialité?}

\section{Éric Méchoulan}

Numéro 3, printemps 2004

Devenir-Bergson

Becoming-Bergson

URI : https://id.erudit.org/iderudit/1005471ar

DOI : https://doi.org/10.7202/1005471ar

Aller au sommaire du numéro

Éditeur(s)

Centre de recherche sur l'intermédialité

ISSN

1705-8546 (imprimé)

1920-3136 (numérique)

Découvrir la revue

Citer cet article

Méchoulan, É. (2004). Bergson anachronique, ou la métaphysique est-elle soluble dans l'intermédialité? Intermédialités / Intermediality, (3), 119-135. https://doi.org/10.7202/1005471ar
Résumé de l'article

À la différence de maints penseurs contemporains, Bergson situe sa pensée dans le cadre explicite de la métaphysique; mais une métaphysique qui n'est plus celle de la substance et de la présence, il s'agit d'une métaphysique de la durée qui implique autant absence que présence. Par là, il est à la fois anachroniquement métaphysicien (obsolète), mais aussi penseur de l'être en tant qu'anachronique. Ce sont ces investissements qui intéressent aujourd'hui l’intermédialité. 


\title{
Bergson anachronique, ou la métaphysique est-elle soluble dans l'intermédialité?
}

\author{
ÉRIC MÉCHOULAN
}

\begin{abstract}
e temps qui passe ne coule pas d'un mouvement linéaire et continu, il L tourne au contraire sur lui-même, générant de petits tourbillons locaux, des courants qui creusent le lit des époques, des formes fluides où une vie sociale se contracte, trouvant dans le repli et le retour un tâtonnement heureux. C'est ainsi que l'on peut comprendre la paradoxale actualité de Bergson dont ni la phénoménologie ni la psychanalyse ni le marxisme ne sont parvenus à nous débarrasser, alors même que chacun semblait bien pointer du doigt les aveuglements de la philosophie bergsonienne et les moyens d'en dépasser les illusions banalement métaphysiques.
\end{abstract}

\section{Le RYTHME (DU) RÉEL}

Comprendre ainsi cette actualité, sur le fond d'une telle conception du temps, c'est d'emblée (un mot qu'il aime) mettre Bergson aujourd'hui dans ce qu'il disait autrefois. En effet, dans son article sur «Le possible et le réel », il renverse l'habitude temporelle qui voit le possible entourer d'une frange d'incertitude le réel comme si nous devions à chaque instant choisir notre action dans un éventail de possibilités : il montre que le possible est suscité à partir de l'action réelle et non le contraire. On pensait que le possible était moins que le réel (puisque l'existence lui fait défaut), en fait, «le possible n'est que le réel avec, en plus, un acte de l'esprit qui en rejette l'image dans le passé une fois qu'il s'est $\operatorname{produit}^{1} »$. Cet acte de l'esprit n'est rien d'autre que la production d'un mirage,

1. Henri Bergson, «Le possible et le réel» [1930], La pensée et le mouvant: Essais et conférences, Paris, Librairie Félix Alcan, 1939 [1922], p. 126-127. Désormais les références à cet ouvrage seront indiquées par le sigle «PR » suivi de la page, et placées entre parenthèses dans le corps du texte. 
le dispositif d'une nécessaire illusion («mirage», «dispositif» sont les termes qu'emploie Bergson) dont rend parfaitement compte un temps verbal : le futur antérieur. Le devenir ne fonctionne donc pas de façon linéaire en allant du possible au réel, mais à rebours, du réel au possible: "c'est le réel qui se fait possible, et non pas le possible qui devient réel» (PR, P. 132; j’ajoute les italiques). Nous n'avons pas affaire à l'inéluctable d'un devenir où, parmi de multiples possibilités, se dessinerait la trajectoire unique du réel, mais une incessante façon de faire advenir le réel en même temps que se fabrique rétrospectivement le possible, comme une interprétation ajoutée à l'événement même. Le devenir-Bergson aujourd'hui doit lui aussi opérer selon ce même dispositif, adoptant la forme d'un faire.

On trouve, dans Les deux sources de la morale et de la religion, un aparté sur la création artistique qui reprend ce modèle temporel du réel et du possible:

Une œuvre géniale, qui commence par déconcerter, pourra créer peu à peu par sa seule présence une conception de l'art et une atmosphère artistique qui permettront de la comprendre; elle deviendra alors rétrospectivement géniale [...]. Dans une spéculation financière, c'est le succès qui fait que l'idée avait été bonne. Il y a quelque chose du même genre dans la création artistique, avec cette différence que le succès, s'il finit par venir à l'œuvre qui avait d'abord choqué, tient à une transformation du goût public opérée par l'œuvre même².

L'œuvre réelle ne se détache pas sur un fond de possibles connus, mais l'artiste invente simultanément une œuvre inattendue et l'attente qu'elle va peu à peu susciter (en ce sens, l'esthétique de la réception et l'herméneutique de l'horizon d'attente sont fondées sur de pieuses illusions ou de mauvaises habitudes). L'œuvre géniale n'est pas simplement celle qui s'impose parmi de nombreux multiples, mais celle qui ouvre tout un champ de possibles rétrospectivement: elle nous force à regarder le passé autrement, donc aussi notre présent différemment. Du coup, c'est bien le passé qui devient surprenant.

La comparaison avec la spéculation financière semble donner au propos bergsonien une tournure banalement pragmatique : la vérité d'une œuvre tiendrait tout entière dans son succès. La seule différence tiendrait à la transformation après-coup du public. Mais une marque s'impose en Bourse parce qu'elle a justement modifié la perception du public en en faisant des acheteurs poten-

2. Henri Bergson, Les deux sources de la morale et de la religion, Paris, Presses universitaires de France, 1961 [1932], p. 75. 
tiels. Il n'y a pas forcément très loin d'une spéculation financière à une spéculation métaphysique: l'action donne un nouveau rythme qui se cristallise en habitude. Comprendre est une activité rétrospective : littéralement, la compréhension tient ensemble deux temps différents, celui du moment où l'œuvre a été créée, où l'événement a eu lieu, moment où l'existence allouée se double d'une projection dans le passé de possibles qui l'auront apparemment permise, et celui des moments où l'événement se reproduit sur la scène sociale sur le mode de l'évidence.

Il est un autre exemple encore plus éclairant sur cette relation entre rythme et compréhension: la lecture, en particulier la lecture à haute voix. Bergson reconnaît bien l'utilité d'une explication de l'œuvre d'un grand écrivain,

encore faut-il que l'élève ait commencé à la goûter, et par conséquent à la comprendre. C'est dire que l'enfant devra d'abord la réinventer, ou, en d'autres termes, s'approprier jusqu'à un certain point l'inspiration de l'auteur. Comment le fera-t-il, sinon en lui emboîtant le pas, en adoptant ses gestes, son attitude, sa démarche? Bien lire à voix haute est cela même. L'intelligence viendra plus tard y mettre des nuances. [...] Avant l'intellection proprement dite, il y a la perception de la structure et du mouvement; il y a, dans la page qu'on lit, la ponctuation et le rythme ${ }^{3}$.

Lire à haute voix conduit donc l'interprète à retrouver, c'est-à-dire à réinventer, le rythme de la composition. Puisque comprendre est avant tout une affaire temporelle où la sensation immédiate du mouvement anticipe sur l'intelligence de l'œuvre à proprement parler, on conçoit que cette anticipation (qui est le double inversé de la rétrospection du possible) prenne la forme d'un rythme $e^{4}$ Goûter et comprendre sont alliés dans la même actualisation d'un rythme réinventé. Et Bergson ajoute:

Soit dit en passant, il y a une certaine analogie entre l'art de la lecture, tel que nous venons de le définir, et l'intuition que nous recommandons au philosophe. Dans

3. Henri Bergson, «Introduction (deuxième partie). De la position des problèmes » [1922], La pensée et le mouvant: Essais et conférerences, p. 108. Désormais les références à cet ouvrage seront indiquées par le sigle «INT » suivi de la page, et placées entre parenthèses dans le corps du texte.

4. Ludwig Wittgenstein fait une remarque du même ordre lorsqu'il avoue n'avoir compris et goûté la poésie de Klopstock qu'à dater du jour où il a découvert les rythmes notés (longues-brèves) qui sous-tendaient l'écriture des vers. (Ludwig Wittgenstein, Leçons et conversations sur l'esthétique, la psychologie et la croyance religieuse, Cyrill Barrett (éd.), trad. Jacques Fauve, Paris, Gallimard, 1971 (1966), p. 21. 
la page qu'elle a choisie du grand livre du monde, l'intuition voudrait retrouver le mouvement et le rythme de la composition, revivre l'évolution créatrice en s'y insérant sympathiquement. (INT, p. 109)

C'est dire que nous devrions plus souvent lire à haute voix Bergson afin de mieux penser l'intuition comme méthode.

\section{L'INTUITION ET L'EXPÉRIENCE DE L'INESSENTIEL}

La méthode philosophique de l'intuition", comme la lecture à haute voix, fonctionne au rythme. L'intuition est insertion dans le rythme même du vivant, dans sa durée propre, de même qu'à sa manière le souvenir interprète la perception afin de pouvoir mieux s'y insérer de façon sympathique ${ }^{6}$. Cependant, 122 l'intuition ne se communique que par l'intelligence: «elle est plus qu'idée; elle devra toutefois, pour se transmettre, chevaucher sur des idées. Du moins s'adressera-t-elle de préférence aux idées les plus concrètes, qu'entoure encore une frange d'images. » (INT, p. 52) Ainsi, la

[métaphysique vraie] commencera par chasser les concepts tout faits; elle aussi [comme la science] s'en remettra à l'expérience. Mais l'expérience intérieure ne trouvera nulle part, elle, un langage strictement approprié. Force lui sera de bien de revenir au concept, en lui adjoignant tout au plus l'image. Mais alors il faudra qu'elle élargisse le concept, qu'elle l'assouplisse, et qu'elle annonce, par la frange colorée dont elle l'entourera, qu'il ne contient pas l'expérience tout entière. (INT, p. 55)

5. Il s'agit bien d'une méthode: insistant sur le fait que «la métaphysique doit procéder par intuition », en prenant garde au «caractère essentiellement actif de l'intuition métaphysique», Bergson poursuit en signalant que «seule la méthode dont nous parlons permet de dépasser l'idéalisme aussi bien que le réalisme.» (Henri Bergson, "Introduction à la métaphysique » [1903], La pensée et le mouvant, p. 233. Désormais les références à cet ouvrage seront indiquées par le sigle «IM » suivi de la page, et placées entre parenthèses dans le corps du texte.)

6. «Le rôle de l'ébranlement perceptif est simplement d'imprimer au corps une certaine attitude où les souvenirs viennent s'insérer. " (Henri Bergson, Matière et mémoire, Paris, Presses universitaires de France, 1985 [1896], p. 108. Désormais les références à cet ouvrage seront indiquées par le sigle «MM» suivi de la page, et placées entre parenthèses dans le corps du texte) «Toute image-souvenir capable d'interpréter notre perception actuelle s'y glisse si bien que nous ne savons plus discerner ce qui est perception et ce qui est souvenir.» (MM, p. 113) Cette interprétation a lieu par contraction des souvenirs: «ce que nous appelons agir, c’est précisément obtenir que cette mémoire 
Cette image de la «frange d'images ${ }^{7}$ » apparaissait déjà dans Matière et mémoire, au moment où Bergson indique que les images-souvenirs débordent par définition la perception susceptible de les accueillir et que le cerveau a pour fonction d'écarter les images qui ne peuvent décidément pas être utilement associées à la perception actuelle: «Tout au plus certains souvenirs confus, sans rapport à la situation présente, débordent-ils les images utilement associées, dessinant autour d'elles une frange moins éclairée qui va se perdre dans une immense zone obscure. » (MM, p. 90) Là où la vie, dans sa dimension intéressée et utilitaire, inhibe les souvenirs, tâchant de limiter au maximum la frange d'images autour de la perception, l'intuition métaphysique cherche, au contraire, à auréoler le concept d'un halo d'images qui lui permettra de garder un contact vivant avec l'expérience concrète de la durée. La frange d'imagessouvenirs est ce qui donne un rythme à la perception comme au concept, elle leur évite de disparaître sous la généralité, pour l'un, ou sous la contingence, pour l'autre. Elle alloue à l'unité du concept ou de la perception la multiplicité de la durée, mais une multiplicité ordonnée: un rythme.

Quoiqu'un rythme se développe dans le temps de façon continue, il le scande, l'accentue, le retourne sur lui-même: dans la continuité même de la durée, il instille des discontinuités. C'est en quoi on ne saurait réduire le continu de la durée chez Bergson à une traditionnelle métaphysique de la substance. L'ontologie de la mémoire, chez lui, permet à la fois de maintenir dans la durée une unité profonde des sujets (la mémoire n'est pas localisée dans un recoin du cerveau, ce sont les êtres qui sont dans la mémoire: d'où la fausse question de rechercher une localisation des souvenirs en général), sans souscrire, pour autant, à une perpétuelle identité à soi-même (au contraire, en faisant du sujet une instance perceptive, vouée à l'actualité et à l'utilité, et une dynamique mémorielle, faite de virtualité et de réflexion, Bergson trouve dans

se contracte ou plutôt s'affile de plus en plus, jusqu'à ne présenter que le tranchant de sa lame à l'expérience où elle pénétrera.» (MM, p. 117)

7. Bergson l'exprime autrement encore dans sa conférence «Introduction à la métaphysique»: "Les divers concepts que nous formons des propriétés d'une chose dessinent donc autour d'elle autant de cercles beaucoup plus larges, dont aucun ne s'applique sur elle exactement.» (IM, p. 212) Lors du colloque de Montréal, une partie de la conférence de Georges Didi-Huberman était justement consacrée à cette notion de frange. Je le remercie d'avoir attiré mon attention sur cette image. Il en développe certains éléments dans un article à paraître: Georges-Didi Huberman, «L'image-sillage », L'inactuel, $\mathrm{n}^{\circ} 10$ [à paraître]. 
chaque instant des sujets un dédoublement perpétuel, ici oublié au profit de l'action, là ressenti pour mieux penser). Le métaphysicien ancien travaillait «sur des concepts déposés par avance dans le langage, comme si, descendus du ciel, ils révélaient à l'esprit une réalité suprasensible. Ainsi naquit la théorie platonicienne des Idées. » (INT, p. 57) Il faut, à l'inverse, que le métaphysicien cher à Bergson voie dans les concepts des mots du vocabulaire quotidien «élaborés par l'organisme social en vue d'un objet qui n'a rien de métaphysique. » (INT, p. 61) Là où l'on croit avoir accès à du suprasensible, on a affaire au découpage social du réel. Il est alors deux options: soit l'on fait confiance au langage ordinaire pour penser les processus signifiants, soit l'on fait confiance à l'expérience ordinaire pour penser l'engendrement du sens. La première voie est celle que suivra Wittgenstein; la seconde, celle qu'emprunte Bergson. Cependant, Bergson apparaît bien proche de Wittgenstein lorsqu'il remarque:

ce ne sont pas des mots que nous apprenons d'abord à prononcer, mais des phrases. Un mot s'anastomose toujours avec ceux qui l'accompagnent, et selon l'allure et le mouvement de la phrase dont il fait partie intégrante, il prend des aspects différents: telle, chaque note d'un thème mélodique reflète vaguement le thème tout entier. (Mм, p. 130-131)

Au delà de cette valeur allouée à l'apprentissage du langage et au contexte rythmique de la phrase, il est, de façon encore plus fondamentale, frappant de voir combien la philosophie offre, pour eux, le même enjeu: redécrire les problèmes métaphysiques afin de montrer qu'il s'agissait de faux problèmes qui sont ainsi, automatiquement, dissous.

En tous les cas, pour Bergson, cela implique de trouver en deçà des mots et des concepts, ou, plus précisément, dans la manière de considérer aussi la frange vaporeuse d'images qui y demeurent collées, une expérience intérieure qu'il appelle intuition. Par l'intuition, on aurait accès non au monde des essences de la métaphysique classique, mais à la durée concrète de l'inessentiel qui auréole d'images-souvenirs les idées. Le vrai penseur, pour Bergson, propose une métaphysique de l'inessentiel. Cette frange constitue une espèce de surcroît et réclame une forme d'inattention: n’entendons pas par là une expérience située en deçà de l'attention (celle que l'esprit prête à la matière, autrement dit l'intelligence), concevons plutôt une expérience cherchant par delà l'attention de l'intelligence un accès immédiat à la pensée: «l'intuition [...] représente l'attention que l'esprit se prête à lui-même, par surcroît, tandis qu'il se fixe sur la matière, son objet. Cette attention supplémentaire peut être méthodiquement cultivée et développée. » (INT, p. 98) 
Car il faut prendre garde à un contre-sens pour Bergson : le cerveau n'a pas "pour fonction de penser, mais d'empêcher la pensée de se perdre dans le rêve; [c'est] l'organe de l'attention à la vie.» (INT, p. 92) Il faut donc que l'intuition, par un notable effort, soit inattentive à la vie matérielle pour devenir attentive à la pensée, pour en faire quelque chose de rêvé ${ }^{8}$. Elle participe moins alors de la compréhension, au sens classique ou herméneutique du terme, que de la mémoire et de la pensée9. Car il y a loin de l'intuition à la paresse : «Nous recommandons une certaine manière difficultueuse de penser. Nous prisons par dessus tout l'effort. [...]: notre intuition est réflexion.»(INT, p. 109) À la

8. «La durée toute pure est la forme que prend la succession de nos états de conscience quand notre moi se laisse vivre, quand il s'abstient d'établir une séparation entre l'état présent et les états antérieurs [...], mais les organise avec lui, comme il arrive quand nous nous rappelons, fondues pour ainsi dire ensemble, les notes d'une mélodie. Ne pourrait-on pas dire que, si ces notes se succèdent, nous les apercevons néanmoins les unes dans les autres, et que leur ensemble est comparable à un être vivant, dont les parties, quoique distinctes, se pénètrent par l'effet même de leur solidarité? » (Henri Bergson, Essai sur les données immédiates de la conscience, Paris, Presses universitaires de France, 1985 [1889], p. 74-75)

9. On pourrait voir dans l'interprétation du film de Rossellini, Europa 51 (1951), par Jacques Rancière une manière de retrouver (malgré la grande différence d'orientation) cette position de Bergson dans le contexte d'une pensée de l'égalité: "personne ne peut voir pour ceux qui ne voient pas, faire savoir de l'ignorance des autres. Le problème n'est pas de savoir ce qu'on fait. Ce savoir-là, quoi qu'en disent les habiles, est le plus ordinairement répandu. Le problème est de penser à ce qu'on fait, de se souvenir de soi [mes italiques]. Au jeune délinquant qu'elle laisse fuir, Irène dit seulement: Pense à ce que tu fais! Il y pensera de fait. Ici la morale de l'histoire et la morale de la caméra s'équivalent: convertir son regard, c'est, au sens strict, pratiquer une nouvelle considération. Le christianisme de l'agnostique Rossellini [...] s'identifie à l'égalité de considération. Cette pratique esthétique et éthique de l'égalité, cette pratique de l'étrangeté égalitaire met en péril tout ce qui est inscrit aux répertoires du social et du politique, tout ce qui représente la société, laquelle ne peut se représenter que sous le signe de l'inégalité, sous la présupposition minimale qu'il y a des gens qui ne savent pas ce qu'ils font et dont l'ignorance impose aux autres la tâche du dévoilement. Or la question n'est pas de dévoiler, elle est de cerner. Le regard d'Irène cerne. L'auréole de la sainteté, c'est d'abord la modestie de ce travail de cerne. » (Jacques Rancière, Courts voyages au pays du peuple, Paris, Éditions du Seuil, coll. «Librairie du xxe siècle», 1990, p. 158-159). L'auréole et le cerne, ici, semblent bien proche de la frange bergsonienne, jusque dans leur fonction anti-herméneutique. L’intelligence dévoile, l’intuition cerne. 
vérité, c'est bien plutôt l'intelligence, lorsqu'elle se fixe dans des habitudes verbales, qui s'avère paresseuse. En quoi? parce qu'elle se fie seulement aux signes qui, par définition, arrêtent la réalité dans l'instant d'une forme: or, «pour penser le mouvement, il faut un effort sans cesse renouvelé de l'esprit. Les signes sont faits pour nous dispenser de cet effort en substituant à la continuité mouvante des choses une recomposition artificielle [...] qui ait l'avantage de se manipuler sans peine ${ }^{10}$. "En épousant le mouvement de la durée, l'intuition relève d'une volonté qui la détourne des opérations matérielles de l'intelligence. Puisqu'elle cherche à agir sur le monde, l'intelligence doit figer artificiellement le mouvement; du coup, elle prend la multiplicité propre aux expériences pour l'application d'idées toutes faites.

Contre ce prêt-à-porter philosophique, Bergson exige de l'intuition qu'elle participe d'un empirisme du sur mesure: «un empirisme digne de ce nom, un empirisme qui ne travaille que sur mesure, se voit obligé, pour chaque nouvel objet qu'il étudie, de fournir un effort absolument nouveau. Il taille pour l'objet un concept approprié à l'objet seul.» (IM, p. 222) Il s'agit là, bien sûr, d'un remarquable paradoxe: comment le concept qui doit, par définition, dire le général pourrait-il épouser de si près la singularité de l'objet? C'est que l'objet est déjà multiplicité; le temps, simultanément actualisation de la mémoire dans la perception singulière et virtualité de tout le passé dans les souvenirs purs: le devenir à l'œuvre dans toute durée suppose sans cesse du nouveau.

Mais c'est aussi ce paradoxe que cherche à éclairer Bergson en en faisant le cheminement propre de ce qu'il entend par métaphysique: «Un empirisme vrai est celui qui se propose de serrer d'aussi près que possible l'original luimême, d'en approfondir la vie, et, par une espèce d'auscultation spirituelle, d'en sentir palpiter l'âme; et cet empirisme vrai est la vraie métaphysique. » (IM, p. 222) L'être en tant qu'être, objet de la métaphysique depuis Aristote, prend chez Bergson les tournures d'un empirisme transcendantal qui ne renie jamais ni la singularité des objets du monde ni leur intégration (au sens mathématique du calcul intégral) dans la règle qui les dépasse: «En ce sens la métaphysique n'a rien de commun avec une généralisation de l'expérience, et néanmoins elle pourrait se définir expérience intégrale.» (IM, p. 255) Pour sentir palpiter l'âme,

10. Henri Bergson, L'évolution créatrice, Paris, Presses universitaires de France, 1986 [1907], p. 328-329. Désormais les références à cet ouvrage seront indiquées par le sigle «EC» suivi de la page et placées entre parenthèses dans le corps du texte. 
il faut la reconnaissance d'un rythme particulier, mais aussi la reconnaissance qu'il y a là du rythme ${ }^{11}$.

Entre l'intuition et l'intelligence, il semble donc bien qu'il y ait une différence de nature. Pourtant, la frange d'images qui entoure le concept témoigne plutôt d'une différence de degré :

Nous avons montré que l'intelligence s'est détachée d'une réalité plus vaste, mais qu'il n'y a jamais eu de coupure nette entre les deux: autour de la pensée conceptuelle subsiste une frange indistincte qui en rappelle l'origine. Bien plus, nous comparions l'intelligence à un noyau solide qui se serait formé par voie de condensation. Ce noyau ne diffère pas radicalement du fluide qui l'enveloppe. Il ne s'y résorbera que parce qu'il est fait de la même substance. (EC, p. 194)

Le rythme désigne justement cette fluidité inessentielle qui enveloppe la détermination intelligente des essences. Pour Bergson, reposer la question métaphysique de l'être en tant qu'être conduit à s'occuper de l'inessentiel. En allant chercher dans la frange d'images qui enveloppe le noyau de l'intelligence, l'accès à l'essence de l'homme ou des objets du monde, il évite les anciennes apories des métaphysiques de la substance sans renoncer à une prise effective sur le monde des essences. Il faut seulement admettre qu'un concept, et même une grammaire des concepts, trouvent leur source dans la fluidité rythmée des événements. La différence de nature n’intervient qu'après-coup, comme un effet rétrospectif de la logique conceptuelle.

À l'inverse du petit choc qui permet parfois de faire cristalliser une solution chimique sursaturée, il faut le petit choc de la volonté pour que l'intuition fluidifie de nouveau le noyau conceptuel. Car percevoir à nouveau la fluidité des images à rebours du solide concept impose un saut, de la même façon (pour reprendre l'exemple qu'utilise Bergson) que la mécanique intellectuelle de la nage n'apprend jamais à nager, si l'enfant ne se décide pas à plonger et à faire l'expérience immédiate des mouvements nécessaires. Différences de degré et différences de nature s'entremêlent ainsi pour donner aux apparences mobiles des stabilités apparentes et au constant devenir les intensités variables du rythme. Pour la métaphysique classique, le devenir n'existe vraiment qu'à se

11. Sur ces points de méthode encore, on pourrait rapprocher Bergson de Wittgenstein. Il n'est qu'à penser, par exemple, aux Bemerkungen über die Farben et à la logique tout à fait empiriste des concepts de couleur que cherche à analyser Wittgenstein. Voir Ludwig Wittgenstein, Remarques sur les couleurs, trad. Gérard Granel, Mauvezin, TransEurop-Repress, 1983 [1977]. 
fondre dans l'éternité d'un donné toujours déjà connaissable, sinon connu; pour la métaphysique renouvelée de Bergson, la durée absolue n'est rien d'autre qu'une incessante création de nouveautés à appréhender (EC, p. 353). Là où l'intelligence lie donc le même au même, l'intuition permet de détourner l'attention vers ces moments sans arrêt singuliers, autres que ce qui était, débordant le noyau solide des concepts. Par une telle attention, l'effort de l'intuition permet de tailler enfin pour chaque objet un concept approprié à lui seul, puisqu'il n'est pas encore complètement détaché de cette frange qui l'enveloppe et lui alloue sa singularité.

\section{LA MÉTAPHYSIQUE DU MONDE OÙ NOUS VIVONS}

128 Les images à l'entour du concept nous ouvrent donc à la pensée de ce que nous sommes, ou plus précisément de ce que nous vivons. Elles légitiment jusqu'au style de Bergson, lui dont l'écriture, d'une élégance inhabituelle pour les philosophes modernes, cherche à redonner aux concepts leurs valeurs singulières à coups de comparaisons et de métaphores qui dilatent l'appréhension des phénomènes: «Ne soyons pas dupes des apparences: il y a des cas où c'est le langage imagé qui parle sciemment au propre, et le langage abstrait qui parle inconsciemment au figuré.» (INT, P. 52) Aux concepts, aux termes abstraits, densifiés dans l'espace, échappe le temps qui, pourtant, les configure. Les termes abstraits sont des métaphores figées: ils nous laissent sur la plage avec un coquillage vide pour comprendre la vague qui l'a traîné jusque-là.

Bergson n'exige pas de nous de renoncer aux mots et aux symboles, il nous demande de voir ce qui les entoure, les permet, les dessine. Au lieu que les expériences se contractent dans des symboles au point de disparaître sous une même écorce, il faudrait pouvoir dilater les mots afin qu'ils rejoignent l'expérience singulière dont ils sont issus. Cette «métaphysique du monde où nous vivons» (INT, p. 54) nous sort d'une herméneutique générale des signes pour nous plonger dans le calcul des engendrements de l'expérience ${ }^{12}$. On doit tâcher d'échapper au "point de vue où l'on risque de rester tant qu'on cherche seulement à comprendre. Mais essayons, en outre, d'engendrer (nous ne le pourrons évidemment que par la pensée).» (INT, p. 77) Par delà la compréhension

12. On peut noter que l'interprétation n'opère pas, chez Bergson, comme le moment indispensable de la compréhension (comme chez Gadamer par exemple), elle ne tient d'ailleurs même pas à un interprète, puisque c'est l'image-souvenir qui interprète la perception afin de pouvoir s'y glisser. 
qui réclame une intelligence des symboles, la pensée doit faire un pas de côté, prêtant attention à ce qui déborde le concept, et se tourner vers le point d'engendrement, l'inflexion particulière du temps, dans lequel s'épanouit soudain une expérience. De nouveau, nous avons affaire au modèle imaginaire du calcul intégral, «calcul des flexions » comme le nommait un de ses inventeurs, Leibniz.

Est-ce à dire que nous voici simplement plongés dans un pur spiritualisme, obstinément individualiste et tout à fait étranger à l'univers social? Rien de moins, puisque l'homme est un être de société et que tout le travail consiste justement à retrouver les points de rebroussement des idées dans la courbe sociale $^{13}$ :

Est proprement humain, en effet, le travail d'une pensée individuelle qui accepte, telle quelle, son insertion dans la pensée sociale, et qui utilise les idées préexistantes comme tout autre outil fourni par la communauté. Mais il y a déjà quelque chose de quasi divin dans l'effort, si humble soit-il, d'un esprit qui se réinsère dans l'élan vital, générateur des sociétés qui sont génératrices d’idées. (INT, p. 76)

13. Là encore, si on voulait trouver une analogie scientifique comme s'y risque souvent Bergson, on dirait que le temps opère selon la logique des systèmes dynamiques non linéaires (le premier travail du jeune professeur Bergson a consisté en une édition de Lucrèce, or la conception épicurienne du monde se fait en fonction d'une physique des fluides où le vortex joue un rôle éminent), où des courbes peuvent changer brutalement de déploiement en fonction de ce qu'on nomme des «attracteurs étranges». Utilisés dans les théories du chaos déterministe, les attracteurs étranges permettent de calculer les comportements chaotiques que l'on trouve en météorologie ou, plus simplement, dans les phénomènes de convection thermique. Dans le cas d'un pendule dont on mesure les oscillations, les deux variables (la vitesse et l'angle du pendule avec la verticale) déterminent une ellipse caractéristique des systèmes dynamiques dissipatifs (c'est-à-dire qui tendent vers l'état de repos où les deux variables sont nulles), cette ellipse est un «attracteur cycle limite» vers lequel tendent toutes les trajectoires issues de n’importe quel point, comme si les points du plan étaient «attirés» vers le point fixe. Mais si l'on ajoute une troisième variable dynamique, par exemple une force extérieure, le régime bipériodique devient chaotique et les trajectoires obéissent à deux impératifs inverses: se contracter selon les principes de la dissipation et s'éloigner en fonction de la «sensibilité aux conditions initiales» (SCI) des forces exercées. Cela génère des «attracteurs étranges», comme disent les mathématiciens qui, en fonction des variables, étirent ou replient les courbes (voir l'attracteur de Lorenz, p. 105). Voir Amy Dahan Dalmédico, Jean-Luc Chabert, Karine Chemla (dirs.), Chaos et déterminisme, Paris, Éditions du Seuil, coll. «Points Sciences», 1992, p. 120-125 et p. 280-282. 
L'intelligence qui permet de comprendre les phénomènes ne suffit jamais, pour Bergson, puisqu'elle nous fait simplement tourner dans la cage des idées toutes faites et des symboles prévisibles. L'outillage mental doit retrouver, à côté, à l'entour des idées, ce vague précis de l'expérience (l'oxymore est nécessaire, mais ne joue pas sur le même plan: vague, du point de vue abstrait des concepts, précis, du point de vue sensible de l'expérience). Le vrai métaphysicien ne s'enfonce donc pas dans la banale pureté des concepts ni dans les profondeurs lumineuses des essences, il tire de côté les idées afin d'en revivre la formation, il les reprend dans le moment où elles apparaissent comme problèmes. Car un des grands propos méthodologiques de Bergson est là : revenir à la formation des vrais problèmes afin de mieux éliminer les faux problèmes générés par l'usage paresseux. Cela implique de redonner à l'immédiateté de l'histoire toute sa valeur. L'écriture de l'histoire ne souscrirait plus à un principe herméneutique de compréhension du passé grâce à la médiation de l'expert historien, mais à une reprise de l'expérience temporelle de la mémoire comme insertion des moments de formation des problèmes dans la création continue des sociétés.

Pensée de la vie contre histoire des formes? Pas exactement. Bien entendu, la forme arrête toujours un processus, de même que le symbole coupe, d'abord, un objet en deux pour, ensuite, le recomposer artificiellement. La forme est le contraire du mouvement et le symbole, le signe d'un faux mouvement. Mais s'occuper de la formation des problèmes indique assez que la question de la forme n'est pas évacuée chez Bergson au profit du simple mouvement vital: rejetant la vertu de l'eidos, qui ouvre toujours, pour lui, sur la forme d'une solution, la genèse des idées dans l'expérience met au jour la formation même des problèmes. De la même façon, on pourrait croire que la focalisation sur l'expérience et ce précaire halo d'images entourant le concept vouerait l'intuition à un piétinement dans la contingence et la rendrait incapable de s'élever au-dessus de l'instant qui l'accueillerait. Bergson n'oublie ni la forme ni l'intelligence: c'est justement l'intelligence qui a pour tâche de pousser l'intuition à dilater l'expérience au delà de l'instinct. L'intelligence est tournée vers la matière et l'espace comme l'instinct vers la vie et la durée : l'intuition est donc bien de l'ordre de l'instinct, mais c'est de l'intelligence qu'est venue la secousse «qui l'aura fait monter au point où elle est. Sans l'intelligence, elle serait restée, sous forme d'instinct, rivée à l'objet spécial qui l'intéresse pratiquement, et extériorisée par lui en mouvements de locomotion.» (EC, p. 179)

Cette dilatation de l'instinct en intuition grâce à la poussée de l'intelligence fait bien en sorte d'agrandir l'instinct sans faire perdre l'ancrage dans 
l'objet lui-même. Simplement, l'instinct, toujours en contact avec le mouvement sensible de l'existence, n'aura plus pour fonction de mouvoir le corps:

C'est à l'intérieur même de la vie que nous conduirait l'intuition, je veux dire l'instinct devenu désintéressé, conscient de lui-même, capable de réfléchir sur son objet et de l'élargir indéfiniment. Qu'un effort de ce genre n'est pas impossible, c'est ce que démontre déjà l'existence, chez l'homme, d'une faculté esthétique à côté [mes italiques] de la perception normale. (EC, p. 178)

Alors même que Bergson lutte contre les néokantiens de son temps, il retrouve ici (jusque dans le vocabulaire des «facultés») la troisième Critique de Kant où l'esthétique servait de lien entre la connaissance de la raison pure et l'action de la raison pratique, justement parce qu'elle avait affaire, de façon libre et désintéressée, au simple jeu des facultés entre elles. Pour Bergson, le philosophe doit s'inspirer de l'artiste et faire le même pas de côté afin d'élargir l'expérience à la «nébulosité vague » (EC, p. 178) où se forment les problèmes, avant qu'ils ne laissent la place à leur dissipation dans l'action ou à leur pétrification dans le concept. D'où la grande modestie de cette métaphysique : l'inessentiel est son domaine, l'à côté son mode opératoire, le nébuleux son recours. Mais l'objectif est encore de parvenir au propre par l'inessentiel, au cour de la vie par l'à côté et à la précision par le nébuleux, afin de mieux éliminer les «fantômes d'idées auxquelles s'accrocheront des fantômes de problèmes.» (EC, p. 179)

\section{LES VERTUS DE L'ANACHRONIQUE}

Pour un penseur de la durée, il paraît sans doute étrange de lui trouver les vertus de l'anachronique. Mais la durée, pour Bergson, est rien moins que cette irréversible flèche du dieu Chronos qui n'atteint jamais sa cible: la durée est faite de plis, de retours, d’à côtés par où le tissu de la vie épouse les mouvements du temps. Par conséquent, l'anachronique déborde sans cesse, enveloppe même la perception du chronique, tout en y glissant subrepticement quelques images-souvenirs bien affûtées.

Ce n'est donc pas parce que l'œuvre de Bergson est manifestement datée et emporte avec elle un cortège de remarques obsolètes et d'obsessions révolues, qu'elle ne pourrait aujourd'hui jouer un rôle de premier plan. Bien entendu, le dialogue que n'a cessé d'entretenir Bergson avec les sciences et les techniques de son temps peuvent encore passer pour un exemple d'attention dans la philosophie qu'on appelle continentale. Ses remarques sur la faculté esthétique et son rapport au sensible ne sont pas forcément éloignées du regain d'intérêt des 
philosophes analytiques pour ces phénomènes. Et l'on pourrait énumérer d'autres possibles présences de Bergson parmi nous. Mais c'est sur la valeur même allouée à l'anachronique que j'aimerais insister. Elle témoigne d'une pensée anti-tragique en général, anti-hégélienne en particulier.

Pour Bergson, la dialectique hégélienne est l'exemple par excellence de l'illusoire médiation et du faux mouvement. Plus encore, c'est tout le travail du négatif qui apparaît comme un fantôme inutile et la puissance du symbole comme un ornement immobile. Freud, dont il aurait pu être bien proche, lui est étranger pour les mêmes raisons: trop tragique. Le tragique durcit toujours les oppositions, il immobilise les destins, il fait croire à un faux dédoublement de l'existence qu'il ramène à la simplicité d'une fin déjà donnée, il alloue à la vie humaine l'apparence grandiloquente et absurde des paralogismes de Zénon. Dans la dilatation de l'expérience que permet l'intuition, Bergson cherche au contraire l'insistance d'une liberté et l'intensité d'un bonheur. Alors que le tragique noue des existences dans de grandes formes où tout commence par la fin, Bergson lâche à demi la proie de l'idée pour l'ombre qui l'enveloppe, afin de faire résonner à chaque fois la mélodie singulière d'une naissance.

L'anachronisme est déjà plié dans ce qui naît, puisque la virtualité du souvenir double l'actualité de la perception. C'est cette non-coïncidence à soimême qui rend possible la dilatation de l'intuition et la fluidité de «l'être en tant qu'être » bergsonien. Le symbole semble, pourtant, l'exemple même de cette dissociation essentielle, dans la mesure où il témoigne d'une coupure originaire. Il s'agit, pour Bergson, d'un faux exemple, car le symbole fige les deux éléments dans un renvoi obligatoire de l'un à l'autre. Rien de moins souple. Si "la métaphysique est donc la science qui prétend se passer de symboles» (IM, p. 206), c'est qu'elle «s'affranchit des concepts raides et tout faits pour créer des concepts bien différents de ceux que nous manions d'habitude, je veux dire des représentations souples, mobiles, presque fluides, toujours prêtes à se mouler sur les formes fuyantes de l'intuition» (IM, p. 213).

C'est en cela que, dans la création continue de l'histoire, l'intermédialité pourrait bien être la continuation de la métaphysique bergsonienne. Il est clair que notre siècle a plutôt consonné avec de farouches prétentions à la destruction ou au dépassement de la métaphysique. Du coup, la tentative bergsonienne de la renouveler semble sans doute un des éléments de sa philosophie les plus irrémédiablement datés. Et pourtant, il ne faut s'illusionner ni sur la soudaine incongruité de la métaphysique ni sur les prétentions à s'en débarrasser. Il est évident que la métaphysique bergsonienne déplace radicalement les enjeux 
classiques de cette philosophie première héritée des Grecs. Sans rechercher, là encore, la grandiloquence des dépassements affectés ou des oublis angoissés, il essaye de donner un tour plus libre, plus souple et plus précis en même temps aux interrogations classiques sur l'être.

Tout le champ des expériences et des temporalités qu'il arpente soigneusement ouvre sur un principe fondamental de continuité. Même s'il faut mettre en scène les différences de nature, à chaque fois, Bergson retrace les différences de degré dans lesquelles elles opèrent. Même si comptent les idées et les représentations, avant tout, il en retrace les contours flottants dans les images qui les enveloppent. En portant l'attention sur l'engendrement des problèmes plutôt que sur la finalité des solutions ou la reproduction des faux problèmes, il oriente les énergies vers la positivité des expériences. En dilatant le regard vers l'à côté et l'inessentiel, il permet de prendre en compte les matérialités troubles de l'histoire comme leur solidification dans des lieux communs ou dans des symboles. Ces divers redéploiements trouvent dans les investigations de l'intermédialité d'identiques principes. Tout y devient affaire de rythme.

Continuation anachronique de la métaphysique bergsonienne, l’intermédialité voudrait donner un tour plus souple aux mots de la tribu, en cherchant autour de l'idée, le bain d'images, d'expériences et de dispositifs techniques dans lequel elle cristallise. Il n'y a pas là recherche de causalismes rapides (que ce soit de la technologie sur les représentations ou des concepts sur les partages sensibles), mais mises en scène des nécessaires fluidités qui font l'expérience la plus commune. On aboutit à un art des situations, dans lequel on voudrait que le vêtement du concept ne flotte pas trop sur le corps de l'expérience.

À la question, donc, de savoir si la métaphysique est soluble dans l'intermédialité, Bergson nous amène à répondre: bien sûr que oui. Et cela tient justement à son anachronisme. Pour un penseur du continu, rien de plus logique au fond que d'avoir des continuateurs (non des disciples). Bergson n'est pas simplement anachronique parce qu'il nous présente aujourd'hui des thèses ou des manières de penser obsolètes (ce qui est juste), il offrait déjà au public de 1900 une mode désuète: le renouvellement de la métaphysique la plus classique. Mais en allant la chercher du côté de Lucrèce plutôt que de Platon, il y faisait fleurir de l'inattendu ${ }^{14}$. Or, c'est bien ce sens du nouveau,

14. Je le rappelais plus haut: le tout premier texte publié par Bergson, dans ses années de professeur de lycée, consiste en des morceaux choisis de Lucrèce (Extraits de Lucrèce, avec un commentaire, des notes et une étude sur la poésie, la philosophie, la 
ce plaisir de l'engendrement qui lui fait donner à la métaphysique une tournure singulière.

L'anachronisme n'est pas seulement cette hérésie historiographique par laquelle on superpose au passé des réflexes du présent (affaire de quantités), mais en fait la constitution même du temps (problème de qualité), ce par où le passé devient chaque fois plus surprenant ${ }^{15}$. En dédoublant chaque instant (actualité de la perception, virtualité du souvenir), Bergson fait du temps qui passe des opérations de subjectivation où l'écart à soi-même est constitutif: l'anachronique fabrique la temporalité autant qu'il la trouble. Ce sont ces petites turbulences qui dessinent les qualités de l'intermédialité : tout ce qui reste et résiste dans le temps et qui forme la durée, cette nappe d'événements asymboliques dans lesquels l'intelligence va façonner des symboles. En ce sens, je ne crois pas que l'on doive lier l'attention aux anachronismes avec la prise en compte de symptômes (qu'ils découvrent un passé occulté ou qu'ils annoncent un futur inconnu), car cette dernière notion nous ramène dans l'orbe de l'herméneutique et d'un principe de dévoilement qui ne permet plus l'intuition du cerne ou de la frange d'images qui entourent le concept. L'intermédialité s'intéresse aux à côtés non à ce qui résiderait au-dessous ou par delà.

L'intermédialité ne résulte donc pas de dispositifs techniques qui constitueraient autant de clés de la production intellectuelle ou de l'invention de sujets qui façonneraient leurs mondes d'objets, mais des contretemps où se contractent les idées et où les événements se dilatent. Autre manière de renouveler l'omniprésence du logos si rapidement condamnée aujourd'hui. Car insister sur l'image ne doit pas immédiatement nous amener à conclure que nous aurions trouvé en Bergson celui qui permet de connaître par l'image plutôt que par le

physique, le texte et la langue de Lucrèce, Paris, Delagrave, 1883). Quoiqu'il n'en parle jamais, on sent bien la présence silencieuse de la pensée épicurienne chez lui : physique des fluides plutôt que des solides, métaphysique du tourbillon et du clinamen plutôt que de l'être. On peut aussi remarquer que les notes de Bergson touchent autant les médiations de la langue et de l'histoire des éditions que l'entrecroisement de la poésie, de la philosophie et de la physique.

15. Voir Nicole Loraux, «Éloge de l'anachronisme en histoire», dans L'ancien et le nouveau, Paris, Le Genre humain/Éditions du Seuil, juin 1993, p. 23-39; Jacques Rancière, «Le concept d'anachronisme et la vérité de l'historien », L'inactuel, nº 6, 1996, p. 53-68; ainsi que la réélaboration de ce concept pour l'histoire de l'art par Georges Didi-Huberman, Devant le temps: Histoire de l'art et anachronisme des images, Paris, Éditions de Minuit, coll. «Critique», 2000. 
langage. Pas plus que la frange d'images qui auréole le concept ne doit impliquer une pensée inconsistante (mais plutôt insistante), le recours à l'intuition ne suppose de renoncer à la précision des opérations de la raison ou du langage. Le dialogue entretenu par Bergson avec les sciences et les techniques de son temps est justement un dialogue, autrement dit un écart respecté, voire revendiqué, et un terrain commun, quelque chose qui court dans les mots (un dia-logos $\left.{ }^{16}\right)$. Le travail de l'intuition sur les images, qui cernent le moment où le concept "prend», libère en fait le concept de son application instantanée dans l'action, il rend perceptible la multiplicité hétérogène qui se décante lentement à la surface du temps pour qu'émerge du nouveau ${ }^{17}$. Sans réduire tout aux propriétés de la langue ou de la rationalité, l'intermédialité prête attention au halo de rêve solidaire des inventions techniques comme des créations idéelles.

16. Le préfixe dia a plusieurs valeurs spatiales ou temporelles: "par, au travers», «pendant que, durant que» ou encore «entre, parmi». Le dialogue n’est pas un résultat ponctuel de l'exercice du logos, il en est inséparable.

17. Cette valeur de l'image serait à rapprocher du travail intérieur à la dialectique hégélienne, puis marxiste, que propose Walter Benjamin, lorsqu'il parle d'image dialectique ou de dialectique au repos. Mais on voit combien Benjamin est forcé de neutraliser le faux mouvement de la dialectique afin de trouver dans la concentration, voire la saturation de l'image l'énergie d'une explosion latente du nouveau. Voir Walter Benjamin, Paris, capitale du XIX siècle. Le livre des passages, trad. Jean Lacoste, Paris, Éditions du Cerf, coll. «Passages», 1993 [1983], p. 478-48o. 